\section{Supplementary text}

\section{Process error for survival and catches}

Survival process is defined by assuming a schooling behavior and schools of equal size but with no temporal variation. This situation corresponds with the concentration of mean survival probability $(\psi)$ approaching infinity in case 3, Table 1 of Mäntyniemi et al. (2015), as follows:

$$
N_{\mathrm{t}+1} \mid N_{\mathrm{t}}, p_{\mathrm{t}}, \psi, s c \sim \operatorname{Betabin}\left(N_{\mathrm{t}}, p_{\mathrm{t}}, \eta,\left(1-p_{\mathrm{t}}\right) \eta\right),
$$

with $\eta=\frac{\left(N_{\mathrm{t}}-s c\right)}{(s c-1)}$, where $s c$ is the group size.
For computation we used the beta approximation to the beta-binomial distribution from Appendix 3 in Mäntyniemi et al. (2015),

$$
s_{\mathrm{t}} \sim \operatorname{Beta}\left(p_{\mathrm{t}} \eta_{\mathrm{t}}^{*},\left(1-p_{\mathrm{t}}\right) \eta_{\mathrm{t}}^{*}\right)
$$

with $\eta_{\mathrm{t}}^{*}=\frac{N(1+\eta)}{(N+\eta)}-1=\frac{N_{\mathrm{t}}}{s c}-1$. To avoid potentially negative values in computation, we assume $\eta_{\mathrm{t}}^{*}=\frac{N_{\mathrm{t}}}{s c}$.

The catch process error is defined in the same way as the survival process, by replacing $p_{\mathrm{t}}$ by $q_{\mathrm{t}}$ and $N_{\mathrm{t}}$ by $d_{\mathrm{t}}$, but not using the beta approximation to beta-binomial distribution.

\title{
JAGS code
}

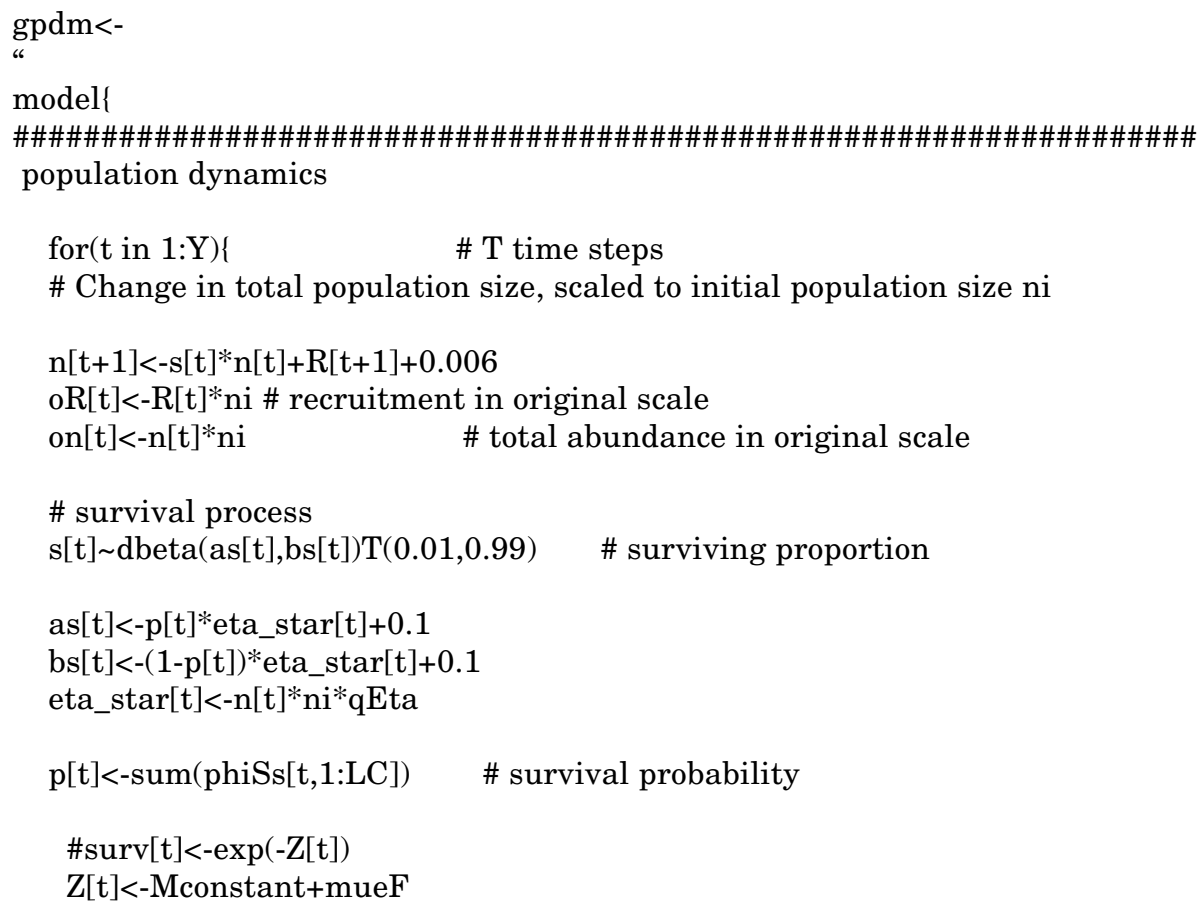




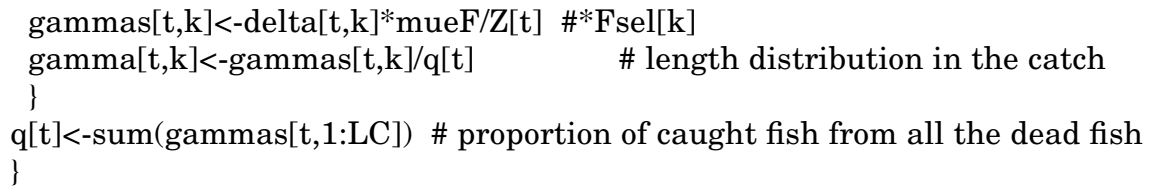

qEta dbeta $(1,1) \mathrm{T}(0.001,0.99)$ \#cluster size as a proportion of the total population size

\#\# Dirichlet prior for the size composition in the beginning of the first time step phi[1,1:LC] ddirch(aphi[1:LC]+0.2)\#aphi[1:LC]\#MUPHI

for $(\mathrm{k}$ in $1: \mathrm{LC})\{$

aphi[k]<-mu_phi[k]*eta_phi

\}

\# Mortality rates and initial abundance: multivariate normal \#prior for a linear combination of transformed parameters

\# -> less correlation between simulated parameters and block updating

\#using M-H alg. -> better mixing

Mconstant<-exp(LM) \# Natural mortality rate

$\mathrm{LM}<-\operatorname{par}[1]+\mathrm{muM}$

Fconstant<-exp(LF) \#Fishing mortality rate

$\mathrm{LF}<-\operatorname{par}[2]+\mathrm{muF}$

$\mathrm{n}[1]<-1$

ni<-exp(lni) \# Initial population size

lni<-par[3]+muN

$R[1]<-0 \quad$ \# no recruits in the first year: they are already

\#included in the initial size distribution

$R[2]<-0.0000000000001$

$\mathrm{R}[3]<-0.0000000000001$

$\mathrm{R}[4]<-0.0000000000001$

$\mathrm{R}[5]<-0.0000000000001$

$\mathrm{R}[6]<-0.0000000000001$

$\mathrm{R}[7]<-0.0000000000001$

$\mathrm{R}[8]<-0.0000000000001$

$\mathrm{R}[9]<-0.0000000000001$

$\mathrm{R}[10]<-0.0000000000001$

$\mathrm{Ja}[1]<-0.0000000000001$

$\mathrm{Jb}[1]<-0.0000000000001$

$\mathrm{Jc}[1]<-0.0000000000001$

$\mathrm{Ja}[2]<-0.0000000000001$

$\mathrm{Jb}[2]<-0.0000000000001$

Jc[2]<-0.0000000000001

$\mathrm{Ja}[3]<-0.0000000000001$

$\mathrm{Jb}[3]<-0.0000000000001$

$\mathrm{Jc}[3]<-0.0000000000001$

$\mathrm{Ja}[4]<-0.0000000000001$

$\mathrm{Ja}[5]<-0.0000000000001$

$\mathrm{Ja}[6]<-0.0000000000001$

$\mathrm{Ja}[7]<-0.0000000000001$

$\mathrm{Jb}[4]<-0.0000000000001$ $\mathrm{Jb}[5]<-0.0000000000001$

$\mathrm{Jb}[6]<-0.0000000000001$

$\mathrm{Jb}[7]<-0.0000000000001$

$\mathrm{Jb}[8]<-0.0000000000001$ 
$\mathrm{Jc}[5]<-0.0000000000001 \# d \operatorname{lnorm}(\log (500), 5.48) \mathrm{T}(0,) ; \# 5.48=1 / \log (1+100 / 500)$

$\mathrm{Jc}[4]<-0.0000000000001 \# d \operatorname{lnorm}(\log (500), 5.48) \mathrm{T}(0$,$) ;$

$\mathrm{Jc}[6]<-0.0000000000001$

$\mathrm{Jc}[7]<-0.0000000000001$

$\mathrm{Jc}[8]<-0.0000000000001$

$\mathrm{Jc}[9]<-0.0000000000001$

Jamean[1]<-0.0000000000001

Jamean[2]<-0.0000000000001

Jamean[3]<-0.0000000000001

Jamean[4]<-0.0000000000001

Jamean[5]<-0.0000000000001

Jamean[6]<-0.0000000000001

Jamean[7]<-0.0000000000001

Jbmean $[1]<-0.0000000000001$

Jbmean[2]<-0.0000000000001

Jbmean[3]<-0.0000000000001

Jbmean[4]<-0.0000000000001

Jbmean[5]<-0.0000000000001

Jbmean[6]<-0.0000000000001

Jbmean[7]<-0.0000000000001

Jbmean[8]<-0.0000000000001

Jcmean[1]<-0.0000000000001

Jcmean[2]<-0.0000000000001

Jcmean[3]<-0.0000000000001

Jcmean[4]<-0.0000000000001

Jcmean[5]<-0.0000000000001

Jcmean[6]<-0.0000000000001

Jcmean[7]<-0.0000000000001

Jcmean[8]<-0.0000000000001

Jcmean[9]<-0.0000000000001

Rmean[1]<-0.0000000000001

Rmean[2]<-0.0000000000001

Rmean[3]<-0.0000000000001

Rmean[4]<-0.0000000000001

Rmean[5]<-0.0000000000001

Rmean[6]<-0.0000000000001

Rmean[7]<-0.0000000000001

Rmean[8]<-0.0000000000001

Rmean[9]<-0.0000000000001

Rmean[10]<-0.0000000000001

for $(\mathrm{t}$ in $(\mathrm{Y}+2):(\mathrm{Y}+5))\{$

$\mathrm{n}[\mathrm{t}] \sim \operatorname{dnorm}(0,1)$

\}

for $(\mathrm{t}$ in $5: 222)\{$

$\mathrm{Cu}[\mathrm{t}]<-\operatorname{Eggs}[\mathrm{t}] * \exp (-\mathrm{li} *(\mathrm{~W}[\mathrm{t}+2,4]+\mathrm{W}[\mathrm{t}+2,3]+\mathrm{W}[\mathrm{t}+2,2]+\mathrm{W}[\mathrm{t}+2,1]+\mathrm{W}[\mathrm{t}+1,4]+\mathrm{W}[\mathrm{t}+1,3]$

$+\mathrm{W}[\mathrm{t}+1,2]+\mathrm{W}[\mathrm{t}+1,1]))$

TCF $[\mathrm{t}]<-\operatorname{step}(\mathrm{T}[\mathrm{t}+1,1]-\mathrm{T}[\mathrm{t}, 4]-0.25) * \operatorname{step}(\mathrm{T}[\mathrm{t}+1,1]-16)$

TCS $[\mathrm{t}]<-\operatorname{step}(\mathrm{T}[\mathrm{t}+1,2]-\mathrm{T}[\mathrm{t}+1,1]-0.25) * \operatorname{step}(\mathrm{T}[\mathrm{t}+1,2]-16)$;

TCT $[\mathrm{t}]<-\operatorname{step}(\mathrm{T}[\mathrm{t}+1,3]-\mathrm{T}[\mathrm{t}+1,2]-0.25) * \operatorname{step}(\mathrm{T}[\mathrm{t}+1,3]-16)$;

TCL $[\mathrm{t}]<-\operatorname{step}(\mathrm{T}[\mathrm{t}+1,4]-\mathrm{T}[\mathrm{t}+1,3]-0.25) * \operatorname{step}(\mathrm{T}[\mathrm{t}+1,4]-16)$; 
\#First juveniles

Jamean $[\mathrm{t}+3]<-\mathrm{TCF}[\mathrm{t}] * \mathrm{Cu}[\mathrm{t}]+\mathrm{TCS}[\mathrm{t}] * \mathrm{Cu}[\mathrm{t}] * \exp (-\mathrm{li} *(\mathrm{~W}[\mathrm{t}+3,1]-\mathrm{W}[\mathrm{t}+1,1])+$

$\left.\mathrm{TCT}[\mathrm{t}]^{*} \mathrm{Cu}[\mathrm{t}] * \exp (-\mathrm{li} * \mathrm{~W}[\mathrm{t}+3,2]+\mathrm{W}[\mathrm{t}+3,1]-\mathrm{W}[\mathrm{t}+1,1]-\mathrm{W}[\mathrm{t}+1,2])-0.01\right)+\mathrm{TCL}[\mathrm{t}]^{*} \mathrm{Cu}[\mathrm{t}]$

$* \exp (-\mathrm{li} *(\mathrm{~W}[\mathrm{t}+3,3]+\mathrm{W}[\mathrm{t}+3,2]+\mathrm{W}[\mathrm{t}+3,1]-\mathrm{W}[\mathrm{t}+1,1]-\mathrm{W}[\mathrm{t}+1,2]-\mathrm{W}[\mathrm{t}+1,3])))$;

Jbmean[t+4]<- Ja[t+3]*r*dnorm((log(Disch[t+4])-4.6052),0,1); \#-179.23, Second juveniles, 179.23 is the mean of Discharges Jcmean $[\mathrm{t}+5]<-\mathrm{Jb}[\mathrm{t}+4] * \mathrm{r} * \operatorname{dnorm}((\log (\operatorname{Disch}[\mathrm{t}+5])-4.6052), 0,1)$; \#]-179.23 Third juveniles

Rmean $[\mathrm{t}+6]<-\mathrm{Jc}[\mathrm{t}+5] * \mathrm{r} * \operatorname{dnorm}((\log (\operatorname{Disch}[\mathrm{t}+6])-4.6052), 0,1)$;

$\mathrm{Ja}[\mathrm{t}+3] \sim \operatorname{dnorm}(\operatorname{Jamean}[\mathrm{t}+3], 0.00000000003) \mathrm{T}(0,) ; \# \operatorname{Jamean}[\mathrm{t}+3]$

$\mathrm{Jb}[\mathrm{t}+4] \sim \operatorname{dnorm}(\mathrm{Jbmean}[\mathrm{t}+4], 0.00000025) \mathrm{T}(0, \mathrm{Ja}[\mathrm{t}+3]) ; \# \mathrm{~B}(3,1)$ is 0

$\mathrm{Jc}[\mathrm{t}+5] \sim \operatorname{dnorm}(\mathrm{Jcmean}[\mathrm{t}+5], 0.00000025) \mathrm{T}(0, \mathrm{Jb}[\mathrm{t}+4])$;

$\operatorname{preR}[\mathrm{t}+6] \sim \operatorname{dnorm}(\operatorname{Rmean}[\mathrm{t}+6], 0.00000025) \mathrm{T}(0, \mathrm{Jc}[\mathrm{t}+5])$;

$\mathrm{ER}[\mathrm{t}+6]<-\log (\operatorname{preR}[\mathrm{t}+6])-0.5 / \mathrm{TR}+\mathrm{eR}[\mathrm{t}+6]$

\#1/TR \# tauer: precision matrix for the recruitment deviations

$R[t+6]<-\exp (E R[t+6])+0.0000001$

$\mathrm{eR}[\mathrm{t}+6] \sim \operatorname{dnorm}(0, \mathrm{TR}) \quad$ \# recruitment residuals

\}

CVR dunif $(0,3)$

$\mathrm{TR}<-1 / \log (\mathrm{CVR} * \mathrm{CVR}+1)$

$\operatorname{par}[1] \sim \operatorname{dnorm}($ mue[1],1/s2M)T(,0.823)

$\operatorname{par}[2] \sim \operatorname{dnorm}(\mathrm{mue}[2], 1 / \mathrm{s} 2 \mathrm{~F}) \mathrm{T}(-0.589$,

$\# \exp (\operatorname{par}[2]+\operatorname{muF}(\log (0.09)))>0.05, \operatorname{par}[2]>\log (0.555)=-0.589$

$\operatorname{par}[3] \sim \operatorname{dnorm}($ mue[3],1/s2N)

mue $[1]<-0$

mue $[2]<-0$

mue $[3]<-0$

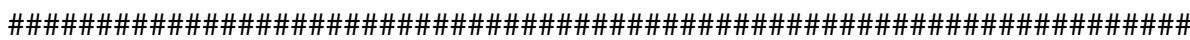

\# Von Bertalanffy Growth model: expected length from size class k

Gk<-Gconstant \# growth rate, fixed for now

Lsigma<-sqrt(Linfsigma*Linfsigma*(1-exp(-Gk*2)))

\# Standard deviation of growths over a time step

\# Linfsigma: standard deviation of lengths of old fish

\# In time, the length distribution of a cohort converges

\# to have mean Linf and SD Linfsigma

for $(\mathrm{k}$ in $1: \mathrm{LC})\{$

Eg[k]<-(Linf-length $[\mathrm{k}])^{*}(1-\exp (-\mathrm{Gk}))+$ length $[\mathrm{k}]$

\}

\#\# Scaled and shifted multivariate logit-normal priors for growth parameters: \#multivariate normal prior for a linear combination of transformed parameters \# -> less correlation between simulated parameters and block updating using \#M-H alg. -> better mixing Linf $<-$ minLinf $+($ maxLinf-minLinf $) *$ pLinf $\operatorname{logit}(\mathrm{pLinf})<-\operatorname{par} 3[1]$ \#dnorm$(20,0.1) \mathrm{T}(15,25)$ \#<-exp(par3[1]) $\operatorname{minLinf}<-18$

$\max \operatorname{Linf}<-20$ 
Linfsigma<-minsdLinf+(maxsdLinf-minsdLinf)*psdLinf

logit(psdLinf)<-par3[3]

minsdLinf $<-0.1$

maxsdLinf $<-3$

Gconstant<-mink+(maxk-mink)*pk

$\operatorname{logit}(\mathrm{pk})<-$ par3[2]

mink $<-0.05 \# 0.1$

$\operatorname{maxk}<-0.08$ \#0.3

$\operatorname{par3[1:3]\sim dmnorm}(\operatorname{mu} 3[1: 3], \operatorname{tau} 3[1: 3,1: 3])$ \# multivariate normal

$\operatorname{mu} 3[1]<-0$

$\mathrm{mu} 3[2]<-0$

$\operatorname{mu} 3[3]<-0$

tau3<-inverse $(\operatorname{cov} 3)$

$\operatorname{cov} 3[1,1]<-1$

$\operatorname{cov} 3[1,2]<-0$

$\operatorname{cov} 3[1,3]<-0$

$\operatorname{cov} 3[2,1]<-\operatorname{cov} 3[1,2]$

$\operatorname{cov} 3[2,2]<-1$

$\operatorname{cov} 3[2,3]<-0$

$\operatorname{cov} 3[3,1]<-\operatorname{cov} 3[1,3]$

$\operatorname{cov} 3[3,2]<-\operatorname{cov} 3[2,3]$

$\operatorname{cov} 3[3,3]<-1$

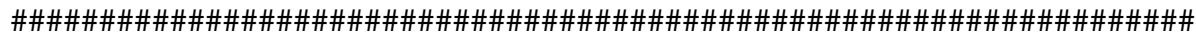

\# Growth matrix: normally distibuted growths from each length class.

\#Negative growths are allowed to counter balance

\# the too fast growths from small sizes. As a result, the size distribution is kept

\#plausible at the population level

\# and the tendency to sink all fish into highest length class becomes avoided.

\# Of course, this is not an appropriate model for individuals

\# I[j]: lower bound of length class $\mathrm{k}$

\# $1[\mathrm{k}]$ : midpoint of length class $\mathrm{k}$

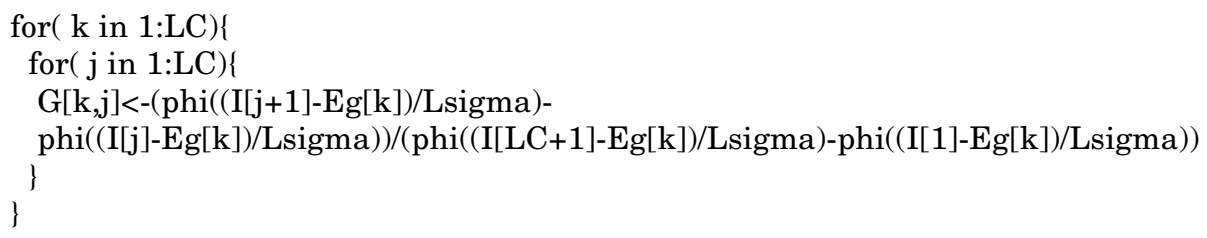

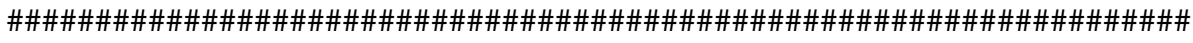

\# weight-length relationship

\# parameters may vary in time, fixed for now

\# for $(\mathrm{t}$ in $1: \mathrm{Y})\{$

\# for $(\mathrm{k}$ in $1: \mathrm{LC})\{$

$\# \log (\mathrm{w}[\mathrm{t}, \mathrm{k}])<-\operatorname{logaw}[\mathrm{t}]+\mathrm{bw}[\mathrm{t}] * \log ($ length $[\mathrm{k}])$

\# \}

\# logaw $[\mathrm{t}]<-$ logawConstant

\#bw $[\mathrm{t}]<-$ bwConstant

$\#$ \}

for $(\mathrm{k}$ in $1: \mathrm{LC})\{$

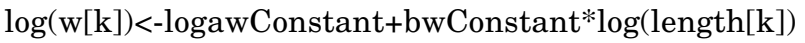


\# Length weight parameters: fixed dummy values for now

logawConstant<--5.84304454 \#ln(0.0029)

bwConstant $<-3.3438$

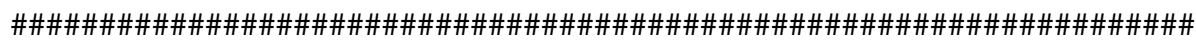

\# Eggs produced by a length class

for $(\mathrm{t}$ in $1: \mathrm{Y})\{$

$\mathrm{SSB}[\mathrm{t}]<-\operatorname{sum}(\mathrm{ssb}[\mathrm{t}, 1: \mathrm{LC}]) / 100 \quad$ \# Calculating SSB for output, not used in the model

$\mathrm{BIOM}[\mathrm{t}]<-\operatorname{sum}($ biom$[\mathrm{t}, 1: \mathrm{LC}])$

for $(\mathrm{k}$ in $1: \mathrm{LC})\{$

eggs $[\mathrm{t}, \mathrm{k}]<-$ mat $[\mathrm{k}] *$ fecConstant*sexr*w[k]

$\mathrm{ssb}[\mathrm{t}, \mathrm{k}]<-\mathrm{mat}[\mathrm{k}]^{*} \mathrm{w}[\mathrm{k}]^{*}$ phiG $[\mathrm{t}, \mathrm{k}]^{*}$ on $[\mathrm{t}]$

\# Spawning stock per length. Not used in the model

\}

biom $[\mathrm{t}, \mathrm{k}]<-\mathrm{w}[\mathrm{k}] *$ on $[\mathrm{t}] *$ phiG $[\mathrm{t}, \mathrm{k}]$

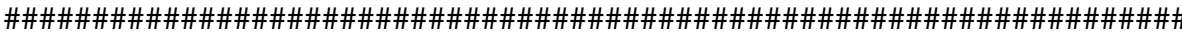

\# total number of eggs in time $t$

for $(\mathrm{t}$ in $1: \mathrm{Y})\{$

Eggs[t]<-inprod(eggs[t,1:LC],phi[t,1:LC] $)^{*} \mathrm{n}[\mathrm{t}]$ \}

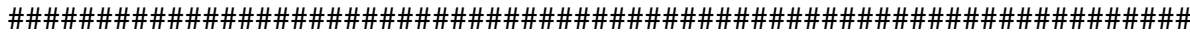

\# Maturity of a length class

for $(\mathrm{k}$ in $1: \mathrm{LC})\{$

mat $[\mathrm{k}]<-$ step(length[k]-matLength)

\}

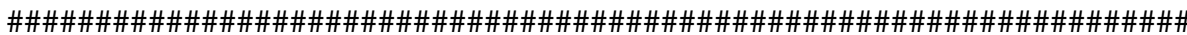

\# fecundity of a length class

\#for(t in 1:Y)

\# for ( $\mathrm{k}$ in $1: \mathrm{LC})\{$

\# fec $[\mathrm{t}, \mathrm{k}]<-$ fecConstant

$\#\}$

$\#\}$

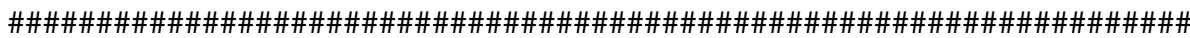

\# sex ratio (females) in a length class

$\#$ for $(\mathrm{t}$ in $1: \mathrm{Y})$;

\#for $(\mathrm{k}$ in $1: \mathrm{LC})$

\# sex $[\mathrm{t}, \mathrm{k}]<-0.5$

\#\}

$\#\}$

sexr $<-0.5$

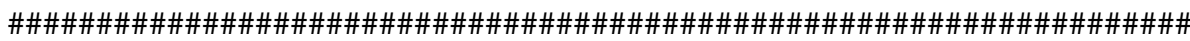

\# Natural mortality

\# for $(\mathrm{t}$ in $1: \mathrm{Y})$ \{

\# for $(\mathrm{k}$ in $1: \mathrm{LC})\{$

\# $\mathrm{M}[\mathrm{t}, \mathrm{k}]<-$ Mconstant \# this will be a function of length

\#\}

$\#\}$

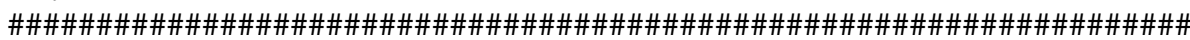

\# Fishing mortality

\#for(t in 1:Y)\{

\#for( $\mathrm{k}$ in $1: \mathrm{LC})\{$

$\# \mathrm{~F}[\mathrm{t}]<-\mathrm{mueF}$

$\#$ 


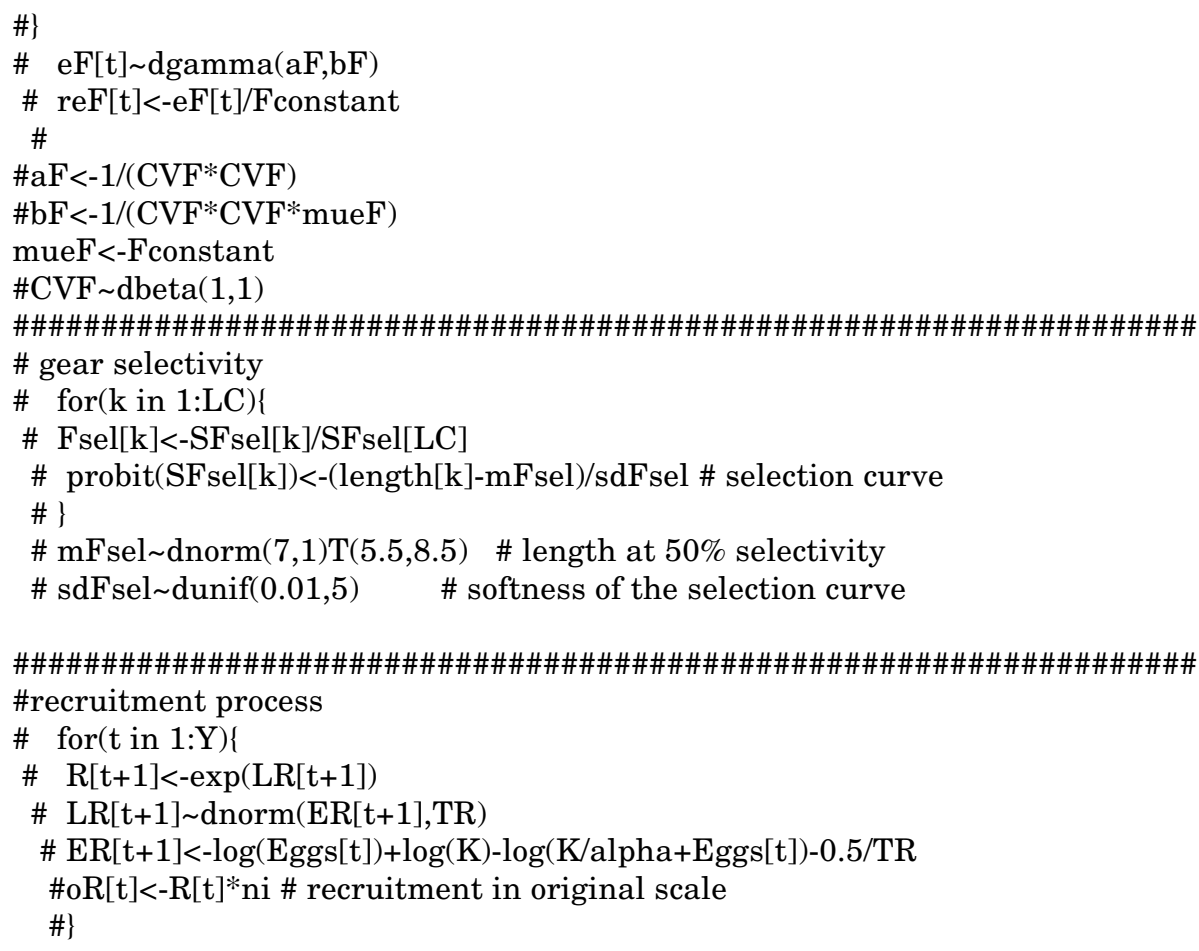

\# Stock recruitment parameters: multivariate normal prior for a linear combination \#of transformed parameters

\# -> less correlation between simulated parameters and block updating using $\mathrm{M}-\mathrm{H}$ alg.

\#-> better mixing

\#oK<-ni* K \# oK : carrying capacity on original scale

$\# \mathrm{~K}<-\exp (\mathrm{LK}) \quad$ \# carrying capacity relative to abundance in 1 st year

\#LK<-par2[1]+mu2[1]

\#logit(alpha)<-Lalpha \# logit normal prior for alpha

\#Lalpha<-par2[1]+mu2[1]-par2[2]-mu2[2]

\#par2[1:2] dmnorm(mue2[1:2],tau2[1:2,1:2])

\#mu2[1]<-muK

$\#$ mu2[2]<-muK-mualpha

\#mue2[1]<-0

\#mue2[2]<-0

\#tau2<-inverse(cov2) \# Covariance matrix to acoount for the transformations \#so that the original parameters

\#cov2[1,1]<-s2K \# still have independent priors

$\# \operatorname{cov} 2[1,2]<-\mathrm{s} 2 \mathrm{~K}$

$\# \operatorname{cov} 2[2,1]<-\operatorname{cov} 2[1,2]$

\#cov2[2,2]<-s2K+s2alpha

\#CVR dunif $(0,1)$ \# CV of recruitment residuals

\#TR<-1/log $(\mathrm{CVR} * \mathrm{CVR}+1)$

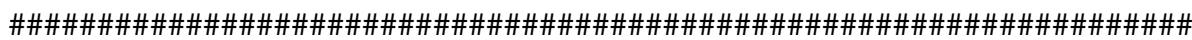

\# fishing process

for $(\mathrm{t}$ in $1: \mathrm{Y})\{$

$\mathrm{d} 1[\mathrm{t}]<-\operatorname{round}\left(\mathrm{n}[\mathrm{t}] *(1-\mathrm{s}[\mathrm{t}])^{*}\right.$ ni $) \quad$ \# number of dead fish

$\mathrm{d}[\mathrm{t}]<-\operatorname{step}(\mathrm{d} 1[\mathrm{t}]-\mathrm{c}[\mathrm{t}]) * \mathrm{~d} 1[\mathrm{t}]+\operatorname{step}(\mathrm{c}[\mathrm{t}]-\mathrm{d} 1[\mathrm{t}]) * \mathrm{c}[\mathrm{t}]$

\# Beta-binomial model for catches 
ac $[\mathrm{t}]<-\mathrm{q}[\mathrm{t}]^{*}$ eta_star $[\mathrm{t}]+0.1$

$\mathrm{bc}[\mathrm{t}]<-(1-\mathrm{q}[\mathrm{t}]) *$ eta_star[t $]+0.1$

$\mathrm{c}[\mathrm{t}] \sim \operatorname{dbetabin}(\mathrm{ac}[\mathrm{t}], \mathrm{bc}[\mathrm{t}], \mathrm{d} 1[\mathrm{t}])$

\# LLC $[\mathrm{t}]<-\operatorname{loggam}(\mathrm{d}[\mathrm{t}]+1)-\operatorname{loggam}(\mathrm{c}[\mathrm{t}]+1)-\operatorname{loggam}(\mathrm{d}[\mathrm{t}]-\mathrm{c}[\mathrm{t}]+1)+$

$\operatorname{loggam}(\mathrm{ac}[\mathrm{t}]+c[t])+\operatorname{loggam}(d[\mathrm{t}]+\mathrm{bc}[\mathrm{t}]-c[\mathrm{t}])-\operatorname{loggam}(\mathrm{ac}[\mathrm{t}]+\mathrm{bc}[\mathrm{t}]+d[\mathrm{t}])+\operatorname{loggam}(\mathrm{ac}[\mathrm{t}]+\mathrm{bc}[\mathrm{t}])$

\#-loggam(ac[t])-loggam(bc[t])

\# pdummy[t]<-step $(\mathrm{d} 1[\mathrm{t}]-\mathrm{c}[\mathrm{t}]) * \exp (\mathrm{LLC}[\mathrm{t}])$

\# dummy[t] dbern(pdummy[t])

\}

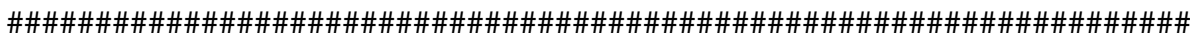

\# Observation model for length data

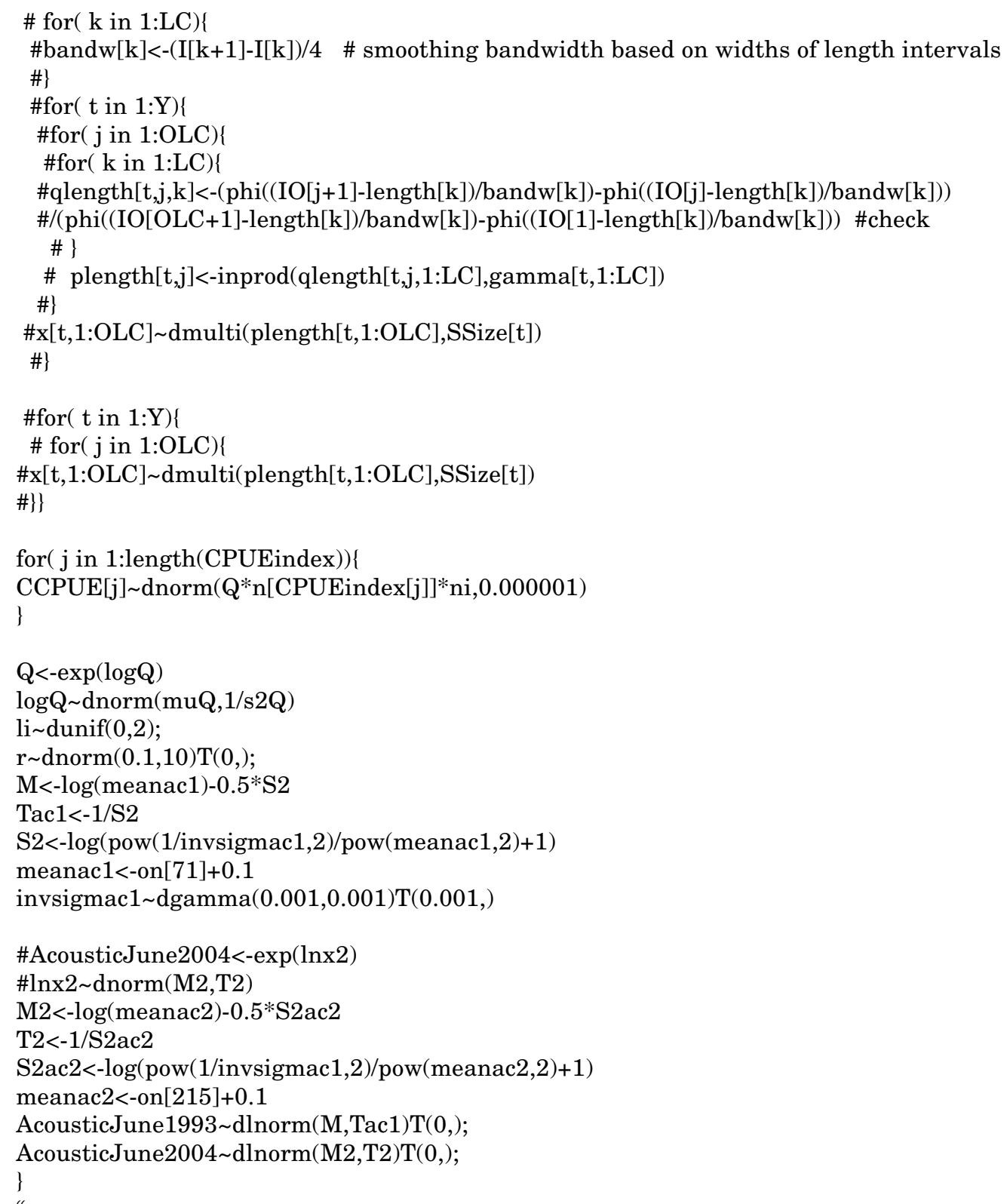

